\title{
Comparação analgésica do Zen Shiatsu e acupuntura auricular em dorsolombalgias de profissionais de enfermagem
}

\section{Analgesic comparison of Zen Shiatsu and auricular acupuncture in back pain among nursing professionals}

\section{Comparación analgésica del Zen Shiatsu y acupuntura auricular en dorsolumbalgias de profesionales de enfermería}

Thaís Dresch Eberhardt'; Lili Marlene Hofstätter ${ }^{I I}$; Sandra Mara Silvério Lopes ${ }^{I I I}$; Edson Antonio Alves da Silva ${ }^{I V}$; Daniela de Cassia Faglioni Boleta Ceranto'; Anair Lazzari Nicola ${ }^{\mathrm{VI}}$

\begin{abstract}
RESUMO: O objetivo deste estudo foi comparar o efeito da aplicação de Zen Shiatsu e de acupuntura auricular na redução dos níveis de dorsolombalgias em profissionais de enfermagem que atuam em ambiente hospitalar. Pesquisa experimental, longitudinal, com análise quantitativa dos dados. A coleta de dados ocorreu em outubro e novembro de 2013, em hospital do Paraná. Foi aplicado um delineamento estatístico completamente casualizado para formar dois grupos com 20 voluntários cada. Os voluntários pertencentes ao grupo 1 receberam uma sessão da intervenção Zen Shiatsu e o grupo 2, uma sessão da intervenção acupuntura auricular. Para mensurar os níveis de dor, foi utilizada a escala visual analógica. Foram comparados os efeitos das duas intervenções com métodos estatísticos não paramétricos, utilizando-se o programa estatístico R para a análise dos dados. Concluiu-se que tanto a acupuntura auricular quanto o Zen Shiatsu mostraram-se igualmente eficazes na redução dos níveis de dorsolombalgias crônicas. Este efeito se manteve por sete dias.
\end{abstract}

Palavras-Chave: Dor nas costas; acupressão; acupuntura auricular; terapias complementares.

\begin{abstract}
This experimental, longitudinal study used quantitative data analysis to compare the effects of Zen Shiatsu and auricular acupuncture in reducing levels of back pain in hospital nursing staff. Data was collected in October and November 2013 at a hospital in Paraná State. Completely randomized statistical design was applied to form two groups of 20 volunteers each. Those in group 1 received a session of Zen Shiatsu and group 2, a session of auricular acupuncture. The visual analogue scale was used to measure pain level. The effects of the two interventions were compared with nonparametric statistical methods using the R statistics program for data analysis. It was concluded that auricular acupuncture and Zen Shiatsu were equally effective in reducing levels of chronic back pain. This effect continued for seven days.

Keywords: Back pain; acupressure; acupuncture, ear; complementary therapies.

RESUMEN: El objetivo de este estudio fue comparar el efecto de la aplicación del Zen Shiatsu y acupuntura auricular en la reducción de los niveles de dorsolumbalgias en profesionales de enfermería que trabajan en un ambiente hospitalario. Investigación experimental, longitudinal, con análisis cuantitativo de los datos. La recolección de datos se llevó a cabo en octubre y noviembre 2013, en hospital de Paraná. Fue utilizado un delineamiento estadístico completamente al azar para formar dos grupos con 20 voluntarios cada uno. Los voluntarios que pertenecían al grupo 1 recibieron una sesión de Zen Shiatsu y, el grupo 2, una sesión de acupuntura auricular. Para medir los niveles de dolor, se ha utilizado la escala visual analógica. Se han comparado los efectos de las dos intervenciones con los métodos estadísticos no paramétricos utilizando el programa estadístico R para el análisis de los datos. Se llegó a la conclusión de que tanto la acupuntura auricular como el Zen Shiatsu se han mostrado igualmente efectivos en la reducción de los niveles de dorsolumbalgias crónicas. Este efecto ha sido mantenido durante siete días.

Palabras Clave: Dolor de espalda; acupresión; acupuntura auricular; terapias complementarias.
\end{abstract}

\section{INTRODUÇÃO}

O trabalho pode ser percebido como fonte de felicidade, reconhecimento, realização e prazer, evidenciado pelo progresso pessoal e econômico propor- cionado aos indivíduos e, também, como causador de desgastes físicos e/ou psíquicos, doenças e acidentes a ele relacionados ${ }^{1}$.

${ }^{I}$ Enfermeira, Especialista em Acupuntura e em Gerenciamento de Enfermagem em Clínica Médica e Cirúrgica, Mestranda do Programa de Pós-Graduação em Enfermagem da Universidade Federal de Santa Maria, Rio Grande do Sul, Brasil. E-mail: thaiseberhardt@gmail.com.

IIEnfermeira, Especialista em Acupuntura, Mestre em Enfermagem Fundamental, Docente do Curso de Enfermagem da Universidade Estadual do Oeste do Paraná. Cascavel, Paraná, Brasil. E-mail:1m hofstatter@yahoo.com.br.

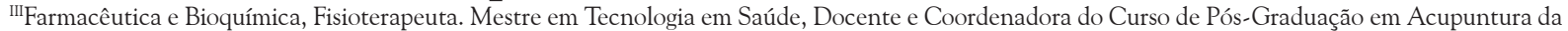
Faculdade de Tecnologia Ibrate. Curitiba, Paraná, Brasil. E-mail: posgraducao@ibrate.edu.br.

${ }^{\mathrm{IV}}$ Licenciado em Física, Doutor em Métodos Numéricos em Engenharia, Docente da Universidade Estadual do Oeste do Paraná. Cascavel, Paraná, Brasil. E-mail: edsonaasilva@gmail.com.

${ }^{v}$ Cirurgiã dentista, Especialista em Acupuntura, Doutora em Odontologia, Professora Titular do Curso de Odontologia da Universidade Paranaense. Cascavel, Paraná, Brasil. E-mail: dcboleta@unipar.br.

vIEnfermeira, Doutora em Enfermagem, Docente do Curso de Enfermagem da Universidade Estadual do Oeste do Paraná. Cascavel, Paraná, Brasil.

E-mail: anairln@yahoo.com.br. 
No Brasil, no ano de 2007, a dor nas costas de causa desconhecida foi a principal causa de invalidez entre aposentadorias previdenciárias e acidentárias, representando uma incidência de 29,96 por 100.000 contribuintes ${ }^{2}$.

Em relação aos trabalhadores da enfermagem em ambiente hospitalar, a dorsalgia e/ou lombalgia são apontados como os distúrbios osteomusculares mais prevalentes ${ }^{3}$ e incapacitantes, tornando-se uma das principais causas de impedimento ou limitação para realização de atividades ${ }^{4,5}$, bem como pela procura por profissionais da área da saúde para resolução do problema ${ }^{5}$.

Tanto a dor aguda quanto a crônica podem ser tratadas com fármacos de prescrição médica, mas podem ser controladas por meio de terapias complementares como a acupuntura, massagens terapêuticas, ioga, entre outros ${ }^{6}$.

Em 2006, o Ministério da Saúde implantou a Política Nacional de Práticas Integrativas e Complementares (PNPIC) no Sistema Único de Saúde (SUS), atuando nos campos da promoção à saúde, prevenção de agravos, manutenção e recuperação da saúde baseada em modelo de atenção humanizada e centrada na integralidade do indivíduo?

Duas terapias complementares que apontam para uma possibilidade terapêutica, na redução do nível de dor, são o Zen Shiatsu ${ }^{8}$ e a auriculoterapia ou acupuntura auricular ${ }^{9}$.10. A partir disso, pergunta-se: ambas as terapias (Zen Shiatsu e acupuntura auricular) promovem redução similar do nível de dorsolombalgias? A hipótese é a de que ambas reduzem, de forma similar, o nível de dorsolombalgias.

Comparar o efeito analgésico das duas terapias se faz necessário, pois são relatados eventos adversos após a aplicação da acupuntura auricular, segundo a literatura, como, por exemplo, dor na orelha, sangramento local, tontura, náuseas e cefaleia. Porém, a frequência de ocorrência desses eventos é rara e não foi relatado nenhum evento adverso grave, de acordo com a literatura ${ }^{11}$. Em relação ao Shiatsu, dois relatos descrevem eventos adversos graves - fratura de $\operatorname{stent}^{12}$ e trombose da veia jugular interna ${ }^{13}$ - após a aplicação da terapia. No entanto, a terapia Zen Shiatsu apresenta outros benefícios, além da analgesia, como melhora da circulação sanguínea, equilíbrio fisiológico e energético ${ }^{8}$.

Além disso, os estudos ${ }^{8-10,14-20}$ não apresentam técnicas uniformes da aplicação das duas intervenções e uniformidade no número de sessões. A partir do conhecimento da aplicação das duas intervenções, do posicionamento do paciente e da melhor intervenção para a redução do nível de dor, os profissionais que aplicam os dois tratamentos podem optar pela melhor técnica para este tipo de patologia.
O objetivo deste estudo foi comparar o efeito da aplicação de Zen Shiatsu e da acupuntura auricular na redução dos níveis de dorsolombalgias em profissionais de enfermagem que atuam em ambiente hospitalar.

\section{REVISÃo DE LITERATURA}

Para esta pesquisa, foi utilizada a definição de dor da International Association for the Study of Pain (IASP), sendo definida como uma experiência sensorial e emocional desagradável associada a dano real ou potencial de tecidos ${ }^{6}$.

Shiatsu é uma técnica de massagem japonesa que consiste na aplicação de pressão dos dedos sobre o corpo. O nome Shiatsu é composto pelas palavras shi, que significa dedo, e atsu, pressão. O Zen Shiatsu é um tipo de técnica de aplicação do Shiatsu, consistindo na aplicação de pressão dos dedos e da palma da mão sobre os principais meridianos do corpo utilizados pela acupuntura, além da movimentação das articulações ${ }^{8}$.

Auriculoterapia é uma técnica da Acupuntura, que usa o pavilhão auricular para efetuar tratamento de saúde, aproveitando o reflexo que a aurícula exerce sobre o sistema nervoso central ${ }^{21: 27}$.

Optou-se por utilizar o termo acupuntura auricular, pois este descreve melhor a técnica empregada, que consiste na aplicação de agulhas com o propósito de estimular o pavilhão auricular ${ }^{22}$.

\section{Metodologia}

Trata-se de um estudo experimental, longitudinal, com análise quantitativa dos dados. A população alvo foi constituída por enfermeiros, técnicos e auxiliares de enfermagem do Hospital Universitário do Oeste do Paraná (HUOP) - vinculado à Universidade Estadual do Oeste do Paraná (UNIOESTE) - que manifestaram sentir dorsolombalgia. Para conhecer o número de profissionais de enfermagem que sentiam este tipo de dor, foi realizada busca ativa através de envio de comunicado interno e visita aos setores de trabalho.

Conforme dados fornecidos pelo Setor de Recursos Humanos da Instituição, trabalhavam, no ano de 2013, 84 enfermeiros, 161 técnicos de enfermagem e 196 auxiliares de enfermagem, totalizando 441 profissionais de enfermagem. Destes, 103 manifestaram sentir dorsolombalgias, representando $23,36 \%$ dos profissionais de enfermagem que trabalhavam na instituição.

Foram incluídos no estudo os profissionais de enfermagem que aceitaram participar da pesquisa e com idade superior a 18 anos, que sentissem dorsalgia, lombalgia ou dorsolombalgias de qualquer origem, aguda ou crônica, que não fizessem uso de medicamentos analgésicos e/ou anti-inflamatórios diariamente. 
Foram considerados critérios de exclusão para a aplicação da acupuntura auricular mulheres em período gestacional ${ }^{23}$ e para a aplicação do Zen Shiatsu presença de hematomas, contusões, erupções de pele e queimaduras na região dorsal, febre, pós-operatório recente, fraturas recentes na coluna, mulheres no primeiro trimestre de gestação, consumo de bebidas alcoólicas e refeição recente.

Foi aplicado um delineamento estatístico completamente casualizado (randomizado) para formar dois grupos com 20 voluntários cada. Para a realização do sorteio, os nomes dos voluntários foram organizados em ordem alfabética e numerados. O sorteio foi realizado mediante o comando sample do programa estatístico $\mathrm{R}^{24}$, associando cada número sorteado a um nome da lista de funcionários participantes.

Os voluntários pertencentes ao grupo 1 (SHI) receberam uma sessão da intervenção Zen Shiatsu e o grupo 2 (AUR), uma sessão da intervenção acupuntura auricular. A coleta de dados ocorreu em outubro e novembro de 2013.

Para a aplicação do tratamento Zen Shiatsu, o voluntário foi posicionado em decúbito ventral e a sequência a seguir foi repetida três vezes ${ }^{8,25,26}$ : pressão com o polegar descendo a primeira linha do Meridiano da Bexiga no dorso, um lado de cada vez; pressão com o polegar descendo a segunda linha do Meridiano da Bexiga no dorso, um lado de cada vez; movimentos circulatórios com o polegar ao redor da escápula, um lado de cada vez; pressão com o polegar descendo a primeira linha do Meridiano da Bexiga no dorso bilateralmente; pressão com o polegar descendo a segunda linha do Meridiano da Bexiga no dorso bilateralmente; pressão com o punho, sobrepondo-se as duas mãos, subindo o Meridiano Vaso Governador ou Du Mai. A localização dos meridianos foi feita conforme Yamamura ${ }^{23}$.

Para a colocação das agulhas semipermanentes, no tratamento acupuntura auricular, após a devida localização dos pontos, foi realizada higienização do pavilhão auricular com algodão e álcool etílico 70\%, aplicação das agulhas esterilizadas afixadas com micropore ${ }^{27}$. $\mathrm{O}$ tamanho de agulhas escolhidas foi de $1,5 \mathrm{~mm}$ de comprimento ${ }^{21}$. Foi solicitado aos indivíduos que mantivessem as agulhas durante sete dias, sem estimulá-las e que comunicassem o pesquisador caso alguma delas caísse.

Os pontos escolhidos foram Shen Men, Rim, Simpático, Analgesia, Relaxamento muscular, Vértebras torácicas e Vértebras lombares. A localização dos pontos foi realizada conforme indicado por Souza ${ }^{21}$.

Para mensurar os níveis de dor, utilizou-se a escala visual analógica (EVA) - escala unidimensional ${ }^{28}$. A EVA foi escolhida, pois existem evidências acerca da sua fidedignidade e validade psicométrica, além de ser mais sensível do que escalas numéricas ou verbais descritivas na mensuração de dor lombar crônica ${ }^{29}$.
O voluntário preencheu, em uma planilha, uma nota de sua dor nos sete dias antes do tratamento, imediatamente após o tratamento e sete dias após, sendo que zero significa ausência de dor e dez a máxima dor sentida. Foram calculadas médias aritméticas dos três momentos de medidas de dor, que foram codificados conforme segue: momento antes - média das notas atribuídas à dor pela EVA nos últimos sete dias antes da aplicação do tratamento; momento pós-imediato - média das notas atribuídas à dor pela EVA imediatamente após a aplicação do tratamento; momento depois - média das notas atribuídas à dor pela EVA sete dias após a aplicação do tratamento.

As aplicações das duas intervenções foram realizadas pelo mesmo terapeuta, que era o pesquisador, sendo ele responsável pela coleta dos dados referentes às medidas de dor.

Foram comparados os efeitos das duas intervenções com métodos estatísticos não paramétricos pela natureza das medidas. Adotou-se o intervalo de confiança de $95 \%$ para os resultados, utilizando-se o programa estatístico $\mathrm{R}^{24}$ para a análise dos dados.

Este estudo foi aprovado pelo Comitê de Ética em Pesquisa envolvendo seres humanos da Universidade Estadual do Oeste do Paraná (UNIOESTE), sob Parecer $n^{\circ}$ 125/2013. Todos os indivíduos foram informados dos objetivos do estudo e assinaram um termo de consentimento livre e esclarecido antes da admissão no experimento. Além disso, a pesquisa atendeu aos aspectos éticos preconizados pela Resolução do Conselho Nacional de Saúde (CNS) n 466 de 2012. Para preservar o anonimato dos participantes da pesquisa, estes foram identificados com números, em ordem crescente, iniciando no número um, conforme o desenvolvimento da coleta de dados.

\section{Resultados e Discussão}

Dos 40 indivíduos selecionados após a randomização, 35 encaixaram-se dentro dos critérios de inclusão e aceitaram participar da pesquisa, sendo 17 do grupo SHI e 18 AUR. Um voluntário do grupo SHI não pôde participar da pesquisa, pois apresentava lesão recente na coluna e os outros quatro foram excluídos da amostra, pois não aceitaram participar da pesquisa.

A amostra possui uma média de idade de 41 anos, variando entre 24 e 58 anos. Esta média de idade é compatível com estudo que encontrou a mesma média entre indivíduos com dor nas costas no sul do Brasi ${ }^{30}$. A maioria dos indivíduos - 33 $(94,29 \%)$ - é do sexo feminino e $2(5,71 \%)$ são do sexo masculino. O que se observa é que a mulher, além de estar inserida no mundo do trabalho, não abandonou suas tarefas domésticas (educação dos filhos, limpeza do lar, pagamento de contas, assistên- 
cia aos familiares enfermos), resultando em dupla ou tripla jornada de trabalho e isto leva a um risco potencial de adoecimento para elas ${ }^{4}$.

As mulheres apresentam maior risco para dor nas costas se comparado aos homens, pois o sexo feminino apresenta algumas características anatomofuncionais - como, por exemplo: menor estatura, menor massa muscular, menor massa óssea, articulações mais frágeis e menos adaptadas ao esforço físico extenuante, maior peso de gordura, que podem contribuir, principalmente, para o surgimento de lombalgias ${ }^{31}$. Portanto, os achados no presente trabalho vêm ao encontro da literatura.

Em relação ao trabalho, os indivíduos possuíam uma média de 15,43 anos de tempo de trabalho na enfermagem, 15 (42,86\%) voluntários atuavam na instituição como auxiliares de enfermagem, 15 (42,86\%) como técnicos de enfermagem e cinco (14,28\%) como enfermeiros.

Quanto às dorsolombalgias, a média de tempo que os indivíduos sentiam este tipo de dor é de 7,86 anos. Entre os voluntários, 17 (48,57\%) sentiam dores de causa desconhecida, $8(22,86 \%)$ relataram ter recebido diagnóstico médico e a causa da dor era relacionada ao trabalho e 10 (28,57\%) possuíam diagnóstico médico, porém com causas diversas de dor.

Estudo indica uma relação positiva entre dor e características sociodemográficas e laborais dos trabalhadores de enfermagem hospitalar ${ }^{32}$.

A região em que mais ocorre dor é a lombar $22(62,86 \%)$-, sendo que dois $(5,71 \%)$ sentem dor somente na região torácica e 11 (31,43\%) em ambas as regiões. Todos os indivíduos sentiam dor crônica, sendo 29 (82,86\%) intermitente e $6(17,14 \%)$ contínua.

Estudo verificou elevada prevalência de distúrbios musculoesqueléticos em técnicas e auxiliares de enfermagem de um hospital público de Salvador - BA $(83,7 \%)$ e encontrou como resultado que a dor em região lombar é a mais presente neste grupo populacional. Este tipo de distúrbio pode estar relacionado às demandas físicas às quais estes profissionais estão mais expostos no seu trabalho, incluindo a realização de movimentos repetitivos com as mãos, adoção da postura em pé e andar a maior parte do tempo, levantamento de carga e realização de muita força muscular ${ }^{3}$.
Um estudo que avaliou a prevalência de lombalgia em profissionais de enfermagem de um hospital de Belo Horizonte - MG em 80 auxiliares, técnicos de enfermagem e enfermeiros observou que ocorrem sintomas musculoesqueléticos em inúmeras regiões corporais, atingindo, principalmente, a região da coluna (lombar, torácica e cervical) ${ }^{3}$.

A maioria dos voluntários - 30 (85,71\%) - relatou que as dorsolombalgias tiveram início quando trabalhavam em setores assistenciais, 3 (8,57\%) eram agricultores, $1(2,86 \%)$ trabalhava em setor administrativo e $1(2,86 \%)$ praticava esportes.

Estudo mostrou que uma das repercussões físicas do trabalhador de enfermagem decorrente da atuação em ressuscitações cardiopulmonares, em enfermarias de clínica médica, é a lombalgia ${ }^{33}$.

Outros autores apontaram que a mobilização no leito, o transporte de pacientes, o banho no leito foram os principais fatores relacionados aos casos de lombalgias entre profissionais de enfermagem de um hospital no Estado do Rio de Janeiro ${ }^{34}$.

Os efeitos das duas intervenções foram realizados aplicando-se o Teste de Correlação de Spearman em três momentos. $\mathrm{O}$ teste mede o grau de correlação entre duas variáveis ${ }^{35}$. Primeiramente, aplicou-se o teste com o objetivo de comparar se as médias dos níveis de dor eram semelhantes entre os grupos. Num segundo momento, aplicou-se o teste pareado, comparando se houve diferença (redução) dos níveis médios de dor, dentro de cada grupo, após a aplicação da intervenção. Num terceiro momento, aplicou-se o teste com o objetivo de comparar se o efeito das duas intervenções foi semelhante.

As médias de dor pela EVA antes e após a aplicação dos diferentes tratamentos e o p-valor obtido após a aplicação do Teste de Correlação de Spearman, com o objetivo de comparar se as médias dos níveis de dor eram semelhantes entre os grupos, são apresentadas na Tabela 1 .

Observa-se que, para todos os níveis de dor (momento antes, pós-imediato e depois), obteve-se p-valor $>0,05$, não sendo significativo para a diferença, ao nível de significância de 5\%.

Os p-valores encontrados após aplicação do Teste de Correlação de Spearman pareado, com-

TABELA 1: Momentos de medidas dos níveis de dor e médias dos níveis de dor, segundo as terapias. Cascavel , PR, Brasil, 2013.

\begin{tabular}{lccc}
\hline \multicolumn{1}{c}{$\begin{array}{c}\text { Momentos de medidas } \\
\text { dos níveis de dor }\end{array}$} & \multicolumn{2}{c}{ Médias dos níveis de dor } & \multirow{2}{*}{ p-valor } \\
\cline { 2 - 3 } Momento antes & Zen shiatsu & Acupuntura auricular & \\
\hline Momento pós-imediato & 5,28 & 5,53 & 0,65 \\
Momento depois & 1,56 & 1,24 & 0,62 \\
\hline
\end{tabular}


parando se houve diferença entre os níveis de dor nos momentos antes e pós-imediato, antes e depois são apresentados na Tabela 2.

Para todas as comparações, foram encontrados $\mathrm{p}$-valores $<0,05$, sendo significativos para a hipótese de diferença, a um nível de significância de 5\%. Ou seja, é possível afirmar que houve redução significativa das médias dos níveis de dor após a aplicação das intervenções e este efeito manteve-se por sete dias.

Por último, aplicou-se o Teste de Correlação de Spearman, testando as médias de diferenças entre os níveis de dor entre os momentos antes e pós-imediato, antes e depois, entre os grupos. Ambos os resultados obtiveram $\mathrm{p}$-valor $>0,05$, não sendo significativos para a hipótese de diferença, a um nível de significância de 5\%, conforme a Tabela 3. Portanto, pode-se inferir que ambas as intervenções tiveram efeitos analgésicos iguais.

Em relação ao Zen Shiatsu, não foram encontrados na literatura resultados semelhantes, em que foi utilizada a mesma metodologia de aplicação da terapia; além disso, nenhum estudo apresenta nível de evidência $1^{36}$. No entanto, estudos que aplicaram técnicas de Shiatsu encontraram resultados significativos na redução da dor.

Um exemplo foi um estudo em que foram aplicadas duas sessões semanais de Shiatsu, durante 16 semanas, em 10 pacientes com síndrome de túnel do carpo, diagnosticados como portadores de distúrbios osteomusculares relacionados ao trabalho (DORT). Foi constatado que houve diferença estatisticamente significativa de alívio de dor e melhora da funcionalidade, porém não houve diferença estatisticamente significativa quando os resultados são comparados aos obtidos mediante terapia convencional ${ }^{14}$.

Uma pesquisa analisou o efeito do relaxamento induzido através do Shiatsu sobre a distribuição de potência das ondas alfa, mediante eletroencefalografia
(EEG), em mulheres com idade até 35 anos. Acredita-se que a presença das ondas alfa esteja relacionada com o estado mental específico, caracterizando a tranquilidade e maior tolerância à dor. Percebeu-se que as áreas frontais e temporais apresentaram um aumento significativo no potencial do padrão alfa, ou seja, os indivíduos que revelaram estas mudanças poderiam apresentar maior tolerância à dor ${ }^{15}$.

Outro estudo descreveu dois casos do uso do Shiatsu, por meio de acupressão em meridiano colateral (Meridiano do Rim), para tratar dor na extremidade do ombro, após colecistectomia laparoscópica. Ambos os pacientes relataram alívio imediato da $\mathrm{dor}^{16}$. Ainda, a massagem com acupressão mostrou-se uma terapia complementar eficaz para diminuição de lombalgia ocupacional em trabalhadores de enfermagem ${ }^{37}$.

Em relação à acupuntura auricular e auriculoterapia, existem estudos com níveis de evidência elevados ${ }^{36}$ na literatura, indicando que a intervenção reduz a dor; no entanto, também apresentam metodologias variadas de aplicação das intervenções.

Uma revisão sistemática e meta-análise acerca dos efeitos da auriculoterapia no manejo da dor concluiu que auriculoterapia é efetiva para o tratamento de uma variedade de tipos de dor, especialmente na dor pós-operatória ${ }^{11}$.

Um estudo experimental aplicou auriculoterapia, com sementes de Vaccaria, em 74 pacientes para dor lombar crônica, utilizando os pontos Shen Men, Subcórtex, Vértebras Lombares. Os resultados encontrados sugerem que a auriculoterapia parece reduzir os níveis de dor lombar crônica ${ }^{17}$. Para o tratamento de DORT, a auriculoterapia também tem se mostrado eficaz na redução dos níveis de dor ${ }^{18}$.

Outros estudos que aplicaram auriculoterapia em idosos com lombalgia também encontraram como

TABELA 2: Comparação dos níveis de dor nos momentos antes, pós-imediato e depois da aplicação das respectivas intervenções. Cascavel, PR, Brasil, 2013.

\begin{tabular}{lcc}
\hline \multirow{2}{*}{ Intervenção } & \multicolumn{2}{c}{ Comparações } \\
\cline { 2 - 3 } & Antes / Pós-imediato & Antes / Depois \\
\hline Zen shiatsu & $<0,0001$ & $<0,0001$ \\
Acupuntura auricular & $<0,0001$ & $<0,0001$ \\
\hline
\end{tabular}

TABELA 3: Médias de diferenças entre os níveis de dor entre os momentos antes, pós-imediato e depois da aplicação das intervenções. Cascavel, PR, Brasil, 2013.

\begin{tabular}{lcc}
\hline \multirow{2}{*}{ Intervenção } & \multicolumn{2}{c}{ Médias de diferenças } \\
\cline { 2 - 3 } & Antes / Pós-imediato & Antes / Depois \\
\hline Zen shiatsu & 3,72 & 3,44 \\
Acupuntura auricular & 4,29 & 3,88 \\
p-valor & 0,401 & 0,638 \\
\hline
\end{tabular}


resultado a redução dos níveis de dor. Entre eles, dois utilizaram pastilhas magnéticas, demonstrando que os efeitos de redução do nível de dor permaneceram por até quatro semanas, após a aplicação da intervenção ${ }^{19,20}$. Também foi confirmado que a auriculoterapia com agulhas pode reduzir a dor lombar em gestantes durante uma semana ${ }^{9}$, apesar de esta ser recomendada com cautela nesse grupo populacional.

Ainda, ao comparar o efeito analgésico da acupuntura auricular, craneoacupuntura de Yamamoto, eletroacupuntura e cinesioterapia no tratamento da lombalgia, conclui que ambas as terapias complementares são semelhantes na redução deste tipo de dor, porém a acupuntura auricular apresenta uma pequena vantagem sobre as demais ${ }^{10}$.

A partir disso, percebe-se que os resultados obtidos na presente pesquisa vão ao encontro dos achados divulgados na literatura. Entretanto, os estudos utilizaram metodologias diversas de aplicação da técnica de acupuntura auricular ou auriculoterapia, com materiais e pontos diversos.

No entanto, um estudo constatou resultado diferente, em que, apesar de a acupuntura auricular apresentar eficácia na redução da dor nas costas, a acupuntura sistêmica mostrou-se mais benéfica ${ }^{38}$.

Considerando que as mulheres possuem maior risco para o desenvolvimento de dor nas costas, sugere-se à instituição que desenvolva alternativas para reduzir as causas ocupacionais para esse problema.

\section{ConClusão}

Tanto a acupuntura auricular - com aplicação de agulhas semipermanentes por sete dias nos pontos Shen Men, Rim, Simpático, Analgesia, Relaxamento muscular, Vértebras torácicas e Vértebras lombares - quanto a técnica de Zen Shiatsu, na metodologia aplicada no estudo, são eficazes na redução dos níveis de dorsolombalgias crônicas, quando utilizadas em profissionais de enfermagem. Este efeito se mantém por sete dias.

Os dois tratamentos obtiveram efeitos similares na redução do nível médio de dor, ou seja, um tratamento não é melhor que o outro.

Uma das limitações deste estudo é que não foi utilizada a medida de dor no momento da aplicação dos tratamentos, pois grande parte dos indivíduos apresentava dor leve ou estava sem dor no momento exato da aplicação terapêutica. Além disso, as mensurações dos níveis de dor não foram realizadas no mesmo horário do dia, não foram consideradas as diferenças de gênero e nem os fatores intervenientes nos limiares de dor dentro do próprio gênero (como o período pré-menstrual); entretanto, o pesquisador que mensurou os níveis de dor conhecia o tipo de intervenção aplicada. Esses fatores caracterizam as fragilidades do estudo.

\section{REFERÊNCIAS}

1.Beck CLC, Prestes FC, Tavares JP, Silva RM, Prochonow AG, Nonnenmacher CQ. Identidade profissional dos enfermeiros de serviços de saúde municipal. Cogitare enferm. 2009;14: 114-9.

2.Meziat Filho N, Silva GA. Disability pension from back pain among social security beneficiaries, Brazil. Rev saúde pública. 2011;45:494-502.

3.Ribeiro NF, Fernandes RCP, Solla DVF, Santos Junior AC, Sena Junior AS. Prevalência de distúrbios osteomusculares relacionados ao trabalho em profissionais de enfermagem. Rev bras epidemiol. 2012;15:429-38. 4.Carvalho LSF, Matos RCS, Souza NVDO, Ferreira REDS. Motivos de afastamento por licença de saúde dos trabalhadores de enfermagem. Cienc cuid saúde. 2010;9:60-6.

5.Schmidt DRC, Dantas RAS. Quality of work life and work-related musculoskeletal disorders among nursing professionals. Acta Paul Enferm. 2012;25:701-7.

6.Kopf A, Patel NP, editores. Guia para o tratamento da dor em contextos de poucos recursos. Seattle (DW): International Association for the Study of Pain; 2010. 7.Ministério da Saúde (Br). Política Nacional de Práticas Integrativas e Complementares no SUS - PNPIC-SUS. Brasília (DF): Ministério da Saúde; 2006.

8.Souza W. Shiatsu dos meridianos: um guia passo a passo. $3^{\text {a }}$ ed. São Paulo: Editora Senac São Paulo; 2010. 9.Wang SM, Dezinno P, Lin EC, Lin H, Yue JJ, Berman MR, et al. Auricular acupuncture as a treatment for pregnant women who have low back and posterior pelvic pain: a pilot study. Am j obstet gynecol. 2009;201:271-9.

10.Mehret MOC, Colombo CCG, Silvério-Lopes S. Estudo comparativo entre as técnicas de a acupuntura auricular, craneoacupuntura de Yamamoto, eletroacupintura e cinesioterapia no tratamento da lombalgia crônica. Rev Bras Terap e Saude. 2010;1:1-12.

11.Asher GN, Jonas DE, Coeytaux RR, Reilly AC, Loh YL, Motsinger-Reif AA, et al. Auriculotherapy for pain management: a systematic review and meta-analysis of randomizes controlled trials. J altern complement med. 2010;16:1097-108.

12.Ichihashi S, Higashiura W, Itoh $\mathrm{H}$, Sakaguchi S, Kichikawa K. Fracture and collapse of ballon-expandable stents in the bilateral common iliac arteries due to shiatsu massage. Cardiovas Intervent Radiol. 2012;35:1500-4. 13. Wada Y, Yanagihara C, Nishimura Y. Internal jugular vein thrombosis associated with shiatsu massage of the neck. J Neurol Neurosug Psychiatry. 2005;76:142-3.

14.Masselli MR, Turatti TF, Cruz CM, Silvestre MR, Pereira JDAS. O Shiatsu como terapêutica alternativa em portadores de distúrbio osteomusculares relacionados ao trabalho. Rev dor. 2010; 11:197-202.

15.Pernambuco CS, Pires VNL, Maulaz MB, Mesquita MG, Caetano LF, Novaes JF, et al. A inferência do shiatsu na distribuição de potência das ondas alfas no córtex cerebral em mulheres adultas. Fit Perf J. 2003; 2:178-82. 16.Yeh C-C, Ko SC, Huh BK, Kuo CP, Wu CT, Cherng CH, et al. Shoulder tip pain after laparoscopic surgery analgesia by collateral meridian acupressure (shiatsu) therapy: a report of 2 cases. J Manipulative Physiol Ther. 2008;31:484-8. 
17.Yeh C-H, Chien L-C, Chiang Y-C, Huang L-C. Auricular point acupressure for chronic low back pain: a feasibility study for 1-week treatment. Evid Based Complement Alternat Med. 2012;.1-9.

18.Araújo APS, Silvério-Lopes S. Auriculotherapy in the treatment of individuals bearing work - related osteomuscular distubances (WRMD)/ repetitive effort injuries (RSI): one retrospective assement. FIEP Bulletin. 2009;79:133-7.

19.Suen LKP, Wong TKS, Chung JWY. Auriculotherapy on low back pain in the elderly. Complement Ther Clin Pract. 2007;13:63-9.

20.Suen LKP, Wong EMC. Longitudinal changes in the disability level of the elders with low back pain after auriculotherapy. Complement Ther Med. 2008;16:28-35. 21.Souza MP. Tratado de auriculoterapia. Brasília (DF): Med Center; 2007.

22.Silvério-Lopes S, Seroiska MA. Auriculoterapia para analgesia. In: Silvério-Lopes S, editor. Analgesia por acupuntura. Curitiba (PR): Omnipax; 2013. p. 1-22.

23.Yamamura Y. Acupuntura tradicional: a arte de inserir. 2ª ed. São Paulo: Roca; 2001.

24.R Core Team. R: A language and environment for statistical computing. Vienna (Austria): R Foundation for Statistical Computing; 2012. [cited in 2015 Apr 12] Available in: http://www.R-project.org/.

25.Jarmey C, Mojay G. Shiatsu: um guia completo. São Paulo: Pensamento; 1991.

26.Martins ES, Lconclli LB. A prática do Shiatsu - na visão tradicionalista chinesa. São Paulo: Roca; 2002.

27.Prado JM, Kurebayashi LFS, Silva MJP. Auriculotherapy effectiveness in the reduction of anxiety in nursing students. Rev esc enferm USP. 2012;46:1200-6. 28.Ministério da Saúde (Br). Cuidados paliativos oncológicos: controle da dor. Rio de Janeiro: INCA; 2001.
29.Souza FAEF, Hortense P. Mensuração da dor. In: Chaves LD, Leão ER, organizadores. Dor: $5^{\circ}$ sinal vital: reflexões e intervenções de enfermagem. $2^{\mathrm{a}}$ ed. São Paulo: Martinari; 2007. p. 99-117.

30.Ferreira GD, Silva MC, Rombaldi AJ, Wrege ED, Siqueira FV, Hallal PC. Prevalence and associated factors of back pain in adults from southern Brazil: a populationbased study. Braz j phys ther. 2011;15:31-6.

31.Silva MC, Fassa AG, Valle NCJ. Dor lombar crônica em uma população adulta do Sul do Brasil: prevalência e fatores associados. Cad saúde pública. 2004;20:377-85. 32. Lima ACS, Magnago TSB, Prochnow A, Ceron MDS, Schardong AC, Scalcon CB. Factors associated with musculoskeletal pain in hospital nursing workers. Rev enferm UERJ. 2014;22:526-32.

33.Sá CMS, Souza NVDO, Lisboa MTL, Tavares KFA. Organização do trabalho e seus reflexos na atuação dos trabalhadores de enfermagem em ressuscitação cardiopulmonar. Rev enferm UERJ. 2012;20:50-5.

34.Hipolito RL, Mauro MIC, Mauricio VC, Mendevil, CL, Silva LA, Gomes SR. A incidência de distúrbios musculoesqueléticos em trabalhadores da equipe de enfermagem em Campos dos Goytacazes. Rev pesqui cuid fundam. 2011;3:2015-23.

35.Vieira S. Bioestatística: tópicos avançados. $3^{\text {a }}$ ed. Rio de Janeiro: Elsevier; 2010.

36.Stetler CB, Morsi D, Rucki S, Broughton S, Corrigan B, Fitzgerald J, et al. Utilization - focused integrative reviews in a nursing service. Appl nurs res. 1998;11:195-206. 37. Borges TP, Kurebayashi LFS, Silva MJP. Occupational low back pain in nursing workers: massage versus pain. Rev esc enferm USP. 2014;48:670-6.

38.Silva E, Silvério-Lopes S. Lombalgia e lombociatalgia: estudo comparativo de tratamento com acupuntura sistêmica e auricular. FIEP Bulletin. 2010;80 (esp). 